\title{
Los Programas de Transferencia de Renta Condicionada bajo la lupa: olvidos, descuidos e invisibilidades
}

\author{
Programas de Transferência Condicional de Renda: \\ esquecimento, descuidos e invisibilidades
}

Conditional Cash Transfer Programs under scrutiny: forgetfulness, carelessness and invisibility

\author{
Dra. Mónica De Martino Bermúdez* \\ Dra. Laura Vecinday Garrido**
}

\begin{abstract}
Resumen: A partir de los setenta, en América Latina, comienzan a procesarse profundos cambios en la matriz de protección social, que alcanzan sus niveles de consolidación, avanzada ya en la década de los noventa. La región in totum se transforma en un laboratorio de especial interés para la implementación de políticas sociales "novedosas" para los países centrales, luego de la caída de los tigres asiáticos. Focalización, descentralización y desconcentración fueron pilares básicos de la nueva ingeniería socio-asistencial. Es así que las Políticas de Transferencia de Renta Condicionada se transformaron en un componente fundamental en la lucha contra la pobreza o, mejor dicho, en su administración y gestión socio-poblacional. Este artículo intenta, en un primer momento, aportar información general sobre tales políticas a nivel de América Latina para, posteriormente, colocar el énfasis en nuestro país - Uruguay. Luego de brindar ese marco teórico conceptual mínimo, desarrollaremos algunos desafíos para pensar la intervención socio-política y asistencial, en el marco de tales políticas y programas.
\end{abstract}

Palabras claves: programas de transferencia de renta; género; familia, pobreza.

Resumo - A partir dos anos setenta, na América Latina, começam a processar-se profundas mudanças na matriz de protecção social, que alcançam níveis de consolidação avançada já na década de 1990. A região in totum se torna um laboratório de especial interesse para a implementação de políticas sociais "inovadoras" para os países centrais após o colapso dos tigres asiáticos. Focalizacão, descentralização e desconcentração foram pilares da nova engenharia de assistência

\footnotetext{
* Profesora Dr ${ }^{\mathrm{a}}$ Titular en Régimen de Dedicación Total en la Universidad de la República (Uruguay). Correspondência: Facultad de Ciencias Sociales - Departamento de Trabajo Social - 4to. Piso. Constituyente 1502. CP 11200. Cordón - Montevideo - Uruguay. Email: <monica.demartino@cienciassociales.edu.uy>

** Profesora Dra Adjunta en Régimen de Dedicación Total en la Universidad de la República (Uruguay). Correspondência: Facultad de Ciencias Sociales - Departamento de Trabajo Social - 4to. Piso. Constituyente 1502. CP 11200. Cordón - Montevideo - Uruguay. Email: <laura.vecinday@cienciassociales.edu.uy>
} 
social. Assim, as políticas de transferência de renda tornaram-se um componente fundamental na luta contra a pobreza, ou melhor, em sua administração sócio-demográfica e gestão. Este artigo busca, em primeiro lugar, fornecer informações gerais sobre essas políticas, no que tange à América Latina, para depositar, posteriormente, a ênfase em nosso país - Uruguai. Depois de fornecer o quadro conceitual mínimo, desenvolveremos alguns desafios para pensar a intervenção político-social e assistencial no âmbito de tais políticas e programas. Palavras-chave: programas de transferência de renda; gênero; família; pobreza.

\begin{abstract}
From the seventies on in Latin America, profound changes in the models of social protection begin to take place, reaching advanced levels of consolidation already in the nineties. Latin America in totum becomes a laboratory for the implementation of "innovative" social policies of special interest to the core countries after the fall of the Four Asian Tigers. Targeting, decentralization and deconcentration were pillars of the new social assistance engineering, placing special emphasis on expected responses from the assisted. In this way, Conditional Cash Transfer Programs became a fundamental component in the fight against poverty, or rather, in its socio-demographic administration and management. This article attempts, first, to provide general information on such policies in Latin America, and then to place emphasis in our country - Uruguay. After providing a minimal conceptual framework, we will develop some challenges to grasp better the socio-political and assistential intervention, within the framework of such policies and programs.
\end{abstract}

Keywords: Income Transfer Programs; gender; family; poverty.

\title{
Introducción
}

En América Latina, a partir del Consenso de Washington, la matriz de protección social avanzó hacia un modelo unificado. El discurso neoliberal, indicando el agotamiento del Estado de Bienestar y la ineficiencia de este en términos de políticas sociales y combate a la pobreza, hizo que la focalización y la apertura a la prestación de servicios vía mercado, además de ser una respuesta pragmática, implicara el repliegue del Estado, que vio recortadas no sólo su participación sino también su responsabilidad en cuanto garantía de las prestaciones básicas. Las nuevas políticas sociales significaron coberturas temporales, escasas y no siempre de calidad. La focalización, justo es decirlo, estuvo y está dirigida a los sectores pobres, especialmente a aquellos en situación de indigencia, bajo la forma de la asistencia pública cada vez más asociada a Programas de Transferencia de Renta Condicionada (PTC), asociados, a su vez, a cambios conductuales de familias e individuos.

Ubicados en la tensión focalización/universalización, protección/ asistencia, ciudadanía/subordinación, los PTC se presentan como expresión de lo que Martínez Barroso (2005) ha denominado asistencialización de la protección social, en la medida que avanzan sobre prestaciones universales pre-existentes, como veremos posteriormente. Basados en el principio de 
distribución de la renta, caro a los Estados de Bienestar, este tipo de programas posee fuertes limitaciones al respecto, que podrían denominarse, en lenguaje parsoniano, como prerrequisitos funcionales para el andamiaje de protección y asistencia social.

Los límites de la distribución pueden ilustrarse considerando, por un lado, los crecientes niveles de concentración de la riqueza en América Latina, de acuerdo a la bibliografía amplia al respecto, y, por otro, los niveles de gastos destinados a los PTC, medidos a través del porcentaje del Producto Bruto Interno (PBI) afectado. Para el caso uruguayo, el Plan de Emergencia Social - del cual hablaremos más tarde - afectó tan solo el $0,6 \%$ del PBI anual y tuvo una cobertura de 102.353 hogares (MIDAGLIA; SILVEIRA, 2011) ${ }^{1}$, mientras el Plan de Equidad - luego presentado - afecta el 0,43\% (MIRZA, LORENZELLI; BANGO, 2010, p. 81). Incluso el propio Banco Mundial señala, en diversos documentos, que en América Latina los costos de este tipo de programa, por ejemplo, no han tenido mayor impacto a nivel fiscal ${ }^{2}$. No obstante, las últimas cifras publicadas por el Ministerio de Desarrollo Social (Mides) indican que entre los años 2004 y 2012, el Gasto Público Social destinado a los sectores más pobres ha crecido a una tasa real anual de $13 \%$.

Más allá de la situación uruguaya, los límites a la distribución de la riqueza son señalados claramente por el Banco Mundial: Ios PTC deben estar focalizados y "ofrecer protección a la mayoría de los individuos y familias que se encuentren expuestos a riesgos sociales y no sean capaces de protegerse a sí mismos"; deben ser consistentes, para no ser ineficientes duplicando cobertura en algunos casos y desprotegiendo a sectores que deberían recibir la prestación; y también deben ser consistentes "con el desarrollo del mercado de trabajo, promoviendo el desarrollo de mecanismos de sostenimiento autónomo por parte de los beneficiarios". Además, el monto de las transferencias debe definirse "dentro de un marco fiscal y social sostenible" (Banco Mundial, s/f).

En dicho documento se establece claramente que el monto de las transferencias debe ser lo suficientemente alto para motivar cambios conductuales en los beneficiarios, pero no tan altos como para desestimular el ingreso al mercado laboral. No es recomendable que la intervención tenga consecuencias sobre las reglas del mercado, es decir, que la población perciba la prestación como un ingreso estable asimilable a un salario. También suele reconocerse que estos programas tienen la virtud de ofrecer beneficios públicos a sectores de población que no tenían un lugar claro en el esquema de protección social clásico centrado en la posición laboral de los sujetos:

\footnotetext{
${ }^{1}$ Mirza, Lorenzelli y Bango (2010, p.81) establecen que el Panes afectó el 1.06\% del PBI.

${ }^{2} \mathrm{Al}$ respecto, ver Las Políticas de Transferencia de Ingresos en Uruguay: Cerrando las brechas de cobertura para aumentar el bienestar. Informe del Banco Mundial (s/f).
} 


\section{ReVistg a}

\} LOS PROGRAMAS DE TRANSFERENCIA DE RENTA - BERMÚDEZ, M. M.; GARRIDO, L. V. \}

DOI: $10.12957 /$ rep.2015.21052

Las prestaciones sociales focalizadas hacia grupos vulnerables de población ocupaban un lugar marginal en el esquema clásico de políticas sociales. Los escasos programas de esa naturaleza tendían a abordar las problemáticas de 'excepción social' - por ejemplo, la infancia abandonada - a lo que se sumaba que una proporción de los mismos se constituían en subsidiarios de los servicios universales, versión de las asignaciones familiares para el sector privado, los servicios de alimentación para trabajadores, entre otros. (MIDAGLIA; SILVEIRA, 2011, p. 230)

Sin embargo, las limitaciones de estos programas son claras en tanto no generan derechos. Sus beneficios se limitan a los valores mínimos de reproducción, profundizan la segmentación de la pobreza y refuerzan los dispositivos de control sobre la misma.

[...] estos programas fueron pensados, diseñados y puestos en funcionamiento para resolver un problema concreto: el costo de un ajuste considerado necesario por quienes tomaron y toman decisiones en cada uno de los países y por los organismos multilaterales de crédito (OMC) también. (PAZ, 2010, p. 8)

Señala Ivo (2011, p. 176) que, según Esping-Andersen, la cuestión de las reformas sociales en América Latina y Europa es bien distinta aunque refiera a los mismos problemas: a) en Europa, el principio de justicia y bienestar no está subordinado al objetivo de eficiencia económica como sí lo estaría en América Latina, donde no surge clara y decididamente la preocupación por maximizar el bienestar; b) Mientras que en Europa adquieren centralidad los patrones demográficos del perfil de los contribuyentes, en América Latina, la preocupación está en el perfil del mercado de trabajo y la escasez de contribuyentes; c) El crecimiento salarial real a largo plazo es uno de los elementos que contribuyen a explicar el desgaste de los sistemas de reparto y de renta en Europa, y en América Latina se observan niveles importantes de empleo formal y una desigualdad extrema en la distribución de la renta.

Los discursos hegemónicos de economistas y políticos presentan estas alteraciones en la matriz protectora como cambios deseables y no como producto de las limitaciones estructurales inherentes al modelo capitalista de desarrollo económico. ${ }^{3}$

\footnotetext{
3 "Ante los fracasos económicos y sociales, una línea interpretativa, ha sido de defensa y reproducción del mismo paradigma de liberalismo social, con pequeños ajustes, en una nueva versión del 'Consenso de Washington Plus'. Así pues, no sería un cambio de paradigma, sino una reconversión ideológica con una cara más humana y eficiente. En definitiva, una adaptación y respuesta ante un nuevo contexto mediante una estrategia para realizar de mejor y más eficiente manera la primacía del mercado sobre la política institucional y social'(BURCHARD, 2004, p. 127), concluyendo que persiste la idea del papel subsidiario, complementario y amortiguador de la política social y el Estado con respecto a los ajustes y requerimientos de estabilización de la economía capitalista." (SERNA, 2007).
} 


\section{Revigta 미 paltg}

\} LOS PROGRAMAS DE TRANSFERENCIA DE RENTA - BERMÚDEZ, M. M.; GARRIDO, L. V.\}

DOI: 10.12957/rep.2015.21052

A partir de tales inquietudes es que se desarrolló el proyecto de investigación titulado Estudio comparado de Programas de Transferencia de Renta Condicionada en Brasil (Bolsa Familia); Argentina (Asignación Universal por Hijo) y Uruguay (Asignaciones Familiares), con el afán de encontrar aquellos aspectos comunes y otros diferenciales que hacen a las propuestas programáticas mencionadas. El presente artículo, de nuestra autoría, indica algunos puntos de reflexión desarrollados por el equipo de investigación del Departamento de Trabajo Social de la Facultad de Ciencias Sociales de la Universidad de la República, en Montevideo. Se trata de las líneas de análisis que caracterizaron la segunda etapa del proyecto, que se indica a continuación.

\section{Propuesta metodológica del proyecto}

Considerando que los PTC en América Latina presentan diversidades y peculiaridades, pero también aspectos convergentes, el proyecto de investigación en el que se basa este artículo contempló dos dimensiones desde el punto de vista metodológico. A saber:

a) Estudio exploratorio de los PTC actualmente en implementación en América Latina. Este estudio se realizó mediante la utilización de dos procedimientos de investigación: (i) relevamiento y análisis de la producción bibliográfica y documental sobre los programas de transferencia de renta en implementación en América Latina, y (ii) aplicación de un cuestionario por internet a los agentes responsables por la coordinación e implementación de cada programa. Ese estudio exploratorio tuvo como objetivo la elaboración de una caracterización general de los PTC en América Latina.

b) Estudio comparativo de los PTCs seleccionados. Inicialmente, los programas de transferencia de renta seleccionados para el desarrollo del estudio comparativo fueron: (i) Brasil (Bolsa Família; (ii) Argentina (Asignación Universal por Hijo), y (iii) Uruguay (Nuevo Régimen de Asignaciones Familiares). El estudio comparativo se realizó a partir de la identificación de ciertos ejes teóricos que configuraron una grilla analítica. A saber: conceptos de pobreza y de familia que subyacen a este tipo de programas; aspectos que los configuran en términos de presupuesto, gestión, condicionalidades y derechos; formas que asume el ejercicio profesional en el marco de tales programas. A partir de tal grilla se analizaron las leyes y documentos institucionales referentes a cada programa.

Obviamente, en el presente artículo, se ordenan algunas reflexiones globales sobre el Nuevo Régimen de Asignaciones Familiares en nuestro país. 


\section{Un panorama general de Uruguay: intentando reeditar la Suiza de América}

Los PTC han sido un instrumento históricamente utilizado en nuestro país, desde épocas tempranas, si lo comparamos con el resto de la región, como mecanismo de protección social ante los riesgos clásicos derivados de las turbulencias en las trayectorias ocupacionales. Generalmente se han tratado de prestaciones de carácter contributivo ancladas en una concepción de necesidad/derecho vinculada a la clase trabajadora. De esa manera, en 1943 se crearon las Asignaciones Familiares (AFAM) destinadas a los trabajadores de la industria y el comercio, las que fueron extendidas luego a los trabajadores rurales y a los desocupados de las ramas mencionadas. En 1960 se amplió el beneficio a los hijos y menores a cargo de jubilados y pensionistas de industria y comercio, rurales, pensionistas a la vejez y empleados públicos (SIENRA, 2007). La creación, en 1967, del Banco de Previsión Social (BPS), con el cometido de coordinar los servicios y las prestaciones y organizar la seguridad social, significó la centralización del sistema. Estas transferencias fueron condicionadas exigiendo la asistencia de los niños y adolescentes al sistema educativo.

Históricamente, las AFAM no incluyeron aquellas familias en cuyo seno no hubiera miembros vinculados a la seguridad social. Las transformaciones acaecidas en el mundo del trabajo han sido el telón de fondo sobre el cual se procesan las alteraciones en el sistema de protección social: la incertidumbre y la flexibilidad en las trayectorias ocupacionales vuelven necesario repensar los mecanismos del seguro y de la asistencia. Ante el crecimiento de la pobreza e indigencia observado en décadas pasadas, la respuesta privilegiada en nuestro país ha sido apelar a los PTC a partir de mecanismos preexistentes como las AFAM, entendidos ahora como programas de combate a la pobreza promovidos por los OMC. ${ }^{4}$

Respecto al panorama uruguayo, existen estudios que alertan sobre la reproducción de las desigualdades sociales. Vigorito et al (2011) abordan la problemática de la igualdad en las dos últimas décadas de nuestra historia, y lo hacen comparando indicadores de ingreso económico con el Índice de Desarrollo Humano. De su análisis deriva que la evolución del bienestar social, tomando solamente los ingresos de los hogares, tuvo pocos logros desde los noventa, debido a la cada vez mayor concentración de los ingresos, al deterioro de los niveles de vida y a la desigual recuperación post crisis del 2002. También en términos de desarrollo humano la evaluación es negativa. Por otro lado, insisten en que no registrándose variaciones sustantivas en el plano de la educación y la salud, las variaciones en el desarrollo

\footnotetext{
${ }^{4}$ La relativamente reciente creación del Mides, tal como ha acontecido en otros países de la región, puede ser interpretada como la expresión institucional de estas transformaciones. La eficiencia de las prestaciones, entendida como una adecuada focalización y la no superposición de recursos (siempre escasos), es uno de los objetivos centrales que se persigue a través de la sofisticación y tecnificación de los dispositivos de intervención social.
} 


\section{Revigta 미 paltg}

\} LOS PROGRAMAS DE TRANSFERENCIA DE RENTA - BERMÚDEZ, M. M.; GARRIDO, L. V. \}

DOI: $10.12957 /$ rep.2015.21052

humano derivarían especialmente de la incidencia de las variables económicas.

Las diferencias entre el universo de este lado de la línea y el universo del otro lado de la línea son tan compactas y se han reforzado tanto - y por ende, naturalizado -, que la pobreza y la miseria aparecen cuando, desde el punto de vista mensurable, los datos son alarmantes (SANTOS, 2010 , p. 1). Cuando esto sucedió, acompañados de la persistente cobertura mediática, Uruguay se despertó de su letargo. Más allá de los esfuerzos actuales en base a PTC, es imposible reeditar el universalismo propio del Uruguay batllista, el país de amplias capas medias y de cercanías sociales, en un panorama social que se caracteriza por la desigualdad y las distancias sociales. Frente a ellas, la respuesta parecería ser una asistencia por veces poco eficiente, una escasa redistribución y un discurso de reconocimiento de derechos y el intento de revivir un universalismo mínimo, estratificado y excluyente.

\section{Breve presentación de los PTC en Uruguay}

Los PTC toman un auge importante en nuestro país a partir de marzo de 2005, con la instalación del primer gobierno de izquierda, el cual colocó especial énfasis en atender las situaciones de vulnerabilidad social. A partir de tal fecha se ejecuta el Plan de Atención Nacional de la Emergencia Social (Panes), que finaliza en diciembre de 2007. El referido plan se proponía atender a la emergencia social generada como consecuencia, fundamentalmente, de los efectos de la crisis del 2002, apuntando muy especialmente a la población ubicada por debajo de la Línea de Indigencia. El Ingreso Ciudadano era el subcomponente principal del Panes, que era otorgado a aquellos que certificaban su situación de casi absoluta falta de ingresos. Otros componentes colocaban énfasis en salidas hacia el mercado de empleo, pero en condiciones endebles y con escasa proyección. Obviamente, salud, educación y alimentación, así como la atención a problemáticas sociales específicas (como refugios para los sin techo) también estaban presentes. ${ }^{5}$

Posteriormente, a partir del 1 de enero de 2008, entra en vigencia el Plan de Equidad Social (PES) como estrategia para la reducción de la pobreza y la desigualdad, teniendo como meta la búsqueda de la equidad en sus variadas dimensiones: social, étnica, de género, etc. Este plan procura abarcar el conjunto de la población - al menos en sus metas - y no solamente aquellos en situación de desventaja social, aunque estos últimos tengan siempre la prioridad. Este principio básico se funcionaliza a partir de la

\footnotetext{
${ }^{5}$ En términos cuantitativos, el Panes provocó la reducción de la tasa de indigencia en un $50 \%$, si tenemos en cuenta que en diciembre de 2004 se ubicaba en un 39\%, y al término del Panes, en un 1.7\%, de acuerdo a Mirza (2010).
} 


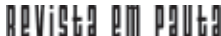

\} LOS PROGRAMAS DE TRANSFERENCIA DE RENTA - BERMÚDEZ, M. M.; GARRIDO, L. V.\}

DOI: 10.12957/rep.2015.21052

consolidación de la dualización del sistema, ya en andamiento desde los noventa, pues consagra la convivencia de prestaciones contributivas y no contributivas. En otras palabras, reúne la lógica del seguro y la de la asistencia para poblaciones cada vez más diferenciadas. ${ }^{6}$

Si bien la premisa fue mantener, en términos de transferencias, ciertos dispositivos históricamente disponibles, como el caso de las AFAM, se realizaron cambios perceptibles en estas. A partir del PES, las modificaciones realizadas a la AFAM pueden resumirse en tres líneas: incremento del monto; feminización del titular y cobro mensual de las prestaciones no contributivas (MIRZA, 2010). En 1995, estas prestaciones fueron limitadas para los trabajadores formales según su nivel de ingreso. En 1999 se aprueba la Ley 17139 , con la cual ingresan al sistema aquellos niños y adolescentes de hogares pobres, sin considerar la condición de trabajador formal de sus integrantes y, por lo tanto, abandona su carácter de prestación contributiva. En 2004, las AFAM se extendieron a las familias con niños y adolescentes de hogares con ingresos menores a tres salarios mínimos.

El ingreso ciudadano previsto en el Panes convivió con un régimen no contributivo de asignaciones familiares hasta la emergencia del PES, cuando las prestaciones fueron unificadas bajo el nombre de AFAM. EI PES significó la reforma del viejo régimen de AFAM y amplió su cobertura a los sectores pobres no indigentes. Si bien las AFAM son el componente sustantivo del PES, han tenido una historia de paulatinas modificaciones, si tenemos en cuenta que en su origen tenían un corte contributivo, como ya hemos visto.

Las AFAM distribuidas por el PES están dirigidas a menores de 18 años pertenecientes a hogares "vulnerables" según indicadores de pobreza. La prestación se vincula con la integración de niños y adolescentes al sistema educativo formal, varía según el número de menores por hogar; por la edad de los mismos y su concurrencia al nivel educativo primario o secundario. Sin embargo, su monto "difícilmente supera el valor del salario mínimo nacional" (MIDAGLIA; SILVEIRA, 2011, p. 236).

Actualmente, es el Banco de Previsión Social el organismo que realiza las prestaciones monetarias establecidas en el PES, luego que en el Panes la responsabilidad fuera del Mides. Las contrapartidas exigidas se vinculan con la inserción en el sistema educativo formal y el cumplimiento de los controles de salud correspondientes.

Midaglia y Silveira indican que las asignaciones familiares, habiendo nacido como un complemento salarial de los trabajadores formales en el ámbito de la seguridad social, se convirtieron en una estrategia de combate a la pobreza: ${ }^{7}$

\footnotetext{
${ }^{6}$ Sobre el PES y los componentes del Panes que se mantuvieron o modificaron, ver Mirza (2010).

${ }^{7} \mathrm{~A}$ julio de 2009, el total de prestaciones del régimen de AFAM alcanzaba a 365.00 niños/as, con una proyección a diciembre del mismo año de unos 450.000 niños/as.
} 
En las versiones en que las Asignaciones se vinculaban con el empleo formal, las obligaciones planteadas contribuían a asegurar una sociedad 'civilizada y garantista', dinámica económicamente, con mecanismos comunes y por ende universales de socialización de los segmentos jóvenes de población [...]. Se asociaban, de alguna manera, al proyecto de una nación moderna, con una mano de obra joven, educada y un mercado de empleo en expansión. Las exigencias establecidas en el nuevo régimen de Asignaciones tienden a cumplir un papel distinto [...] buscan incidir en las condiciones de vida de los estratos sociales desfavorecidos. (2011, p. 243-244)

Las reflexiones de algunos agentes políticos uruguayos alertan sobre algunos riesgos relativos a los PTC: la no inclusión en el mercado de trabajo de la población beneficiaria ante el temor de perder las prestaciones; no poder superar un formato de mero amparo a la pobreza; dificultades en los canales de comunicación con la población beneficiaria; y los límites estructurales en términos de una economía dependiente fuertemente condicionada, lo que coloca constreñimientos a la expansión de la inversión social (MIRZA, 2010).

En un estudio coordinado por Mirza, Lorenzelli y Bango (2010, p. 18) se señala que estos programas "adquieren en su mayor parte los recursos a través del financiamiento internacional, básicamente del banco Mundial o del BID". Allí también se señala que estos organismos han sido los principales promotores de los PTC. ${ }^{8}$

Con matices, los PTC han proliferado en el momento histórico en el que varios países del continente son gobernados por la izquierda política. La opinión hegemónica favorable a estos programas de combate a la pobreza, compartida por gobiernos de izquierda y organismos internacionales como el Banco Mundial, no deja de resultar curiosa, considerando las históricas desavenencias entre estos actores.

\section{Sobre algunos límites que derivan de la ingeniería política}

En este ítem desarrollamos lo que para nosotras son algunos límites de la concepción y diseño de tales programas. Los señalaremos a seguir, a modo de subtítulos.

\section{Algunas precisiones teóricas sobre capital humano}

La categoría "capital humano" es la base del arsenal teórico que orienta la formulación e implementación de los PTC. La ingeniería política de estos dispositivos encuentra allí sus fundamentos y principios normativos.

${ }^{8} \mathrm{Al}$ respecto, ver también Draibe (2006) y Draibe y Riesco (2009). 


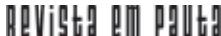

\} LOS PROGRAMAS DE TRANSFERENCIA DE RENTA - BERMÚDEZ, M. M.; GARRIDO, L. V.\}

DOI: 10.12957/rep.2015.21052

Capital humano, en sus múltiples expresiones, se encuentra asociado a otros constructos teórico-metodológicos, como lo es la Escuela de la Elección Racional. Realizaremos algunas precisiones teóricas al respecto que deben ser tenidas en cuenta a la hora de pensar los PTC como dispositivos de intervención social.

La subordinación del homo sociologicus al homo economicus que implicó el surgimiento de la teoría de la Elección Racional en la década de los ochenta (BECKER, 1976; COLEMAN, 1990; OLSON, 1965), se presenta de una manera nueva y sorprendente en la racionalidad que alienta a los PTC (DE MARTINO, 2009; 2012).

Los abordajes teóricos que nos preocupan aparecen como una expresión refinada para capturar los diversos aspectos de la vida social, desde aquellos más públicos como otros más vinculados a la vida privada (de posición política hasta tasas de natalidad). Son constructos que proponen explicar el comportamiento social y político partiendo de dos premisas: que las personas poseen un capital social, siendo posible invertirlo y desarrollarlo, y que esto se logra gracias a conductas racionales. La racionalidad inherente a la conducta humana es una premisa básica en estos enfoques. Detengámonos en dos de los problemas teóricos que se desprenden de estos abordajes y de la racionalidad de los PTC.

\section{La distinción entre actuar como si fuese racional y actuar racionalmente}

Parecería que de estos programas se desprende que la agencia social es entendida como si las personas actuasen racionalmente de manera confirmada y comprobada empíricamente. ¿Qué se tiene en mente cuando se habla de cambios conductuales a cambio de una prestación? ¿O de una prestación que generará cambios conductuales? Refiere a que el modelo que resulta de las perspectivas de los PTC permite previsiones precisas sobre las acciones de las personas y sobre los efectos de sus acciones.

Detrás de este razonamiento está la proposición epistemológica de que la validez de una premisa teórica depende de su poder de previsión. Sin embargo, como sabemos, la fuerza de una teoría no depende de su poder de predicción. Dada la complejidad de la sociedad, la corroboración o falsación de una teoría no son relevantes porque ellas pueden ser debidas a otras mediaciones que pueden potencialmente intervenir (LAWSON, 1989). Por otro lado, de la observación entre el modelo teórico y la realidad, se podría legítimamente inferir que las personas generalmente actúan de acuerdo con principios básicos de racionalidad, pero sería un error tomar tal congruencia como evidencia empírica de que las personas normalmente actúan racionalmente. Cabe señalar, además, que muchas veces las opciones, si bien racionales, no están vinculadas a un proyecto de vida 
global, sino que responden a situaciones coyunturales. Este punto es importante, pues las elaboraciones sobre capital humano parecería que apelan a la capacidad de proyección racional de conductas que disminuyan los impactos o las situaciones de riesgo en el interjuego Estado, mercado y grupos familiares (DE MARTINO, 2009).

Cabe destacar que este tipo de abordaje teórico no toma en cuenta enteramente el contexto cultural en el cual las personas se encuentran insertas o lo que ellas consideran como convicción fundada en la razón. Trabaja con convicciones sobre la relación entre acción y resultado, lo que necesariamente se apoya en nociones culturales que muchas veces no son la de los sujetos a los cuales se "aplican" sus presupuestos teóricometodológicos. Los esfuerzos de familias y hogares para superar barreras socio-económicas, diríamos para acumular capital social, muchas veces son infructuosos ante tales impedimentos, que son de índole claramente estructural. Entonces, ¿cuál es el papel que le cabe a la comprobación empírica de cambios conductuales "exitosos"? Otras veces el círculo de la pobreza/exclusión hace que ni se intenten acciones racionales para poder salir del mismo. Nos encontramos frente a otra racionalidad: la guiada por la impotencia, que, claro, se encuentra vinculada a dimensiones culturales y psicológicas en el marco de un modelo de desarrollo que multiplica la desigualdad económica y la inequidad política (DE MARTINO, 2009).

\section{Cuando evaluación se confunde con control informático}

Es sugerente que, en el campo del PES, el Mides haya creado una Unidad de Seguimiento con alcance nacional, encargada de verificar el cumplimiento de las condiciones exigidas para su inclusión en los programas. Se basa en dispositivos que incluyen: visitas domiciliarias para los nuevos ingresos al régimen de AFAM, elaboración de los correspondientes informes y otros procedimientos que permitan identificar a aquellos hogares que, reuniendo las condiciones, no acceden a las prestaciones (MIRZA, 2010).

Maximizar el acceso a los beneficios y evitar duplicación de prestaciones, parecería que impiden reflexionar sobre la aplicación de las nuevas tecnologías de información al ámbito de lo social, como forma de "mapear" la pobreza, conocerla minuciosamente en sus aspectos cada vez más individualizados para gestionarla de tal manera que continúe siendo dócil bajo el amparo del "reconocimiento" de algunos derechos.

La tecnificación creciente de los sistemas de información en la gestión de lo social se inscribe como parte de la modalidad de asistencia gerencial caracterizada por Grassi (2003) y responde a la necesidad creciente de identificar, contar y clasificar a los usuarios de la asistencia. La racionalidad eficientista, característica de los 90, exigió que, además de la 


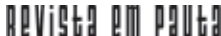

\} LOS PROGRAMAS DE TRANSFERENCIA DE RENTA - BERMÚDEZ, M. M.; GARRIDO, L. V. \}

DOI: 10.12957/rep.2015.21052

prestación de un servicio social, el mismo - en un contexto de "recursos escasos" fuera eficiente, para lo cual se requería individualizar a sus beneficiarios para desarrollar estrategias específicas de atención que proporcionaran lo estrictamente necesario para atender cada problema particular (DE MARTINO, VECINDAY, 2011).

En ese sentido, y teniendo como telón de fondo las transformaciones de las formas de intervención sociopolítica, importa la observación de Castel (1984, p. 143), quien, al analizar un dispositivo de asistencia francés, señala que no es casual que la infancia se haya constituido como preocupación prioritaria porque "[...] con la voluntad de constituir un banco completo de datos sobre la infancia se lleva a cabo también el proyecto de controlar las contingencias y planificar incluso las deficiencias para conseguir un programa de gestión racional de las poblaciones".

Podríamos abordar este aspecto de los PTC como una expresión tecnificada de la asistencialización de las políticas sociales: registro de pobres, control sobre los pobres, control sobre las prestaciones, como si la chances de superación de la pobreza fueran mínimas. El flujo poblacional que deambula por los barrios periféricos y por las agencias sociales se encuentra perfectamente identificado y se informa habitualmente sobre su situación.

\section{Limitaciones que derivan de decisiones definicionales: de las necesidades a la clasificación de los asistidos}

Las pretensiones moralizantes sobre el comportamiento de los pobres se expresan en el tipo de contrapartidas exigidas y en las limitaciones de la mercantilización de la asistencia. Valga, a modo de ilustración, la imposibilidad de comprar bebidas alcohólicas y refrescantes con el uso de la tarjeta alimentaria del Mides. Sin embargo, para otorgar la prestación no es suficiente la medición por ingresos. La exclusión de población para el acceso a los programas se realiza por ciertos criterios de clasificación. El nivel de focalización alcanzado es reconocido por la literatura sobre los PTC. Medir la indigencia y/o la pobreza significó la construcción de indicadores "objetivos" que permitieron la inclusión/exclusión de los beneficiarios de sus prestaciones. De este modo, dentro de la propia ingeniería institucional de los PTC, queda consagrado su carácter de prestación que exige la clasificación de los asistidos y, por ende, también se convalida que no estamos frente a la afirmación de un derecho a la asistencia. En el caso del Panes, se construyó el Índice de Carencias Críticas para la selección de los hogares beneficiarios. Dicho índice capturaba información sobre ingresos junto con una aproximación a estos a través de variables de difícil manipulación por parte de los individuos. A modo de ejemplo, dentro del algoritmo construido para las áreas urbanas (AMARANTE et al., $\mathrm{s} / \mathrm{f}$ ) señalamos 


\section{Revigta 미 paltg}

\} LOS PROGRAMAS DE TRANSFERENCIA DE RENTA - BERMÚDEZ, M. M.; GARRIDO, L. V. \}

DOI: 10.12957/rep.2015.21052

algunos de los indicadores utilizados: ${ }^{9}$ presencia de un funcionario púbico o jubilado/pensionista en el hogar; acceso a cobertura privada de salud; "riqueza" (entendida como la posesión de electrodomésticos más comunes o aquellos más sofisticados, acceso a algunos servicios de recreación como la televisión para abonados, etc.); clima educativo, es decir, el promedio de años de estudio de los adultos del hogar; otros indicadores más clásicos como hacinamiento, tipos de vinculación a la red de saneamiento; tipo de propiedad o uso de la vivienda, etc..

Parecería que el apelo a este tipo de indicadores e índices dejan traslucir cierto "encantamiento" (SOUSA, 2010) por su capacidad de sintetizar información, evaluaciones y perfil de los beneficiarios en apenas un número. Pero su uso merece también algunas precisiones: además de sintetizar, pueden ocultar situaciones relevantes para las políticas sociales, ya que trabajan con medias, promedios que desdibujan situaciones sociales particulares. Parecería que estos indicadores vienen sustituyendo el concepto por la medida creada para operativizarlo, de tal manera que si el indicador (basado en promedios) no varía, no varía lo que se quiere medir. Además, son ciegos a la hora de analizar las relaciones de poder tanto del aparato público como de los propios beneficiarios (SOUSA, 2010, p. 240-241).

Sin embargo, nos interesa abordar este informacionalismo del que habla Castells (1999), como expresión de otro tipo de mutación. Antes, las políticas sociales propias del universalismo estratificado uruguayo se basaban en necesidades, especialmente de las masas trabajadoras protegidas a partir de una malla contributiva. La protección social, entendida como seguridad social, instituyó derechos que atendieron a diversas necesidades sociales de los trabajadores, que se generaban tanto por el tipo de patrón de acumulación como por las características típicas de las diferentes etapas de la trayectoria vital y familiar. Era la hora de los conflictos en torno a la redistribución de la riqueza más que la hora del reconocimiento de derechos (FRASER, 1997).

Actualmente, la ingeniería política de la protección y asistencia social, estratifica a los individuos, reconoce derechos diferenciados - protección contributiva y asistencia no contributiva -, consagra la desigualdad, paradójicamente a partir del reconocimiento de derechos para los más pobres entre los pobres. Frente a los trabajadores asalariados, protegidos por una naturaleza contractual productiva, encontramos un creciente número de "inválidos coyunturales" (DONZELOT, 1986) o de supernumerarios (CASTEL, 1998). Si a la protección típica del Estado de Bienestar le correspondía una ciudadanía universal, aunque abstracta, al tiempo histórico de los PTC le corresponde una ciudadanía regulada, en lo que a protección contributiva refiere, y otra invertida, en lo que hace a asistencia no

${ }^{9}$ Los indicadores fueron diferenciales según áreas (urbana, rural) (AMARANTE et al., s/f). 


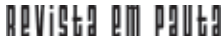

\} LOS PROGRAMAS DE TRANSFERENCIA DE RENTA - BERMÚDEZ, M. M.; GARRIDO, L. V.\}

DOI: 10.12957/rep.2015.21052

contributiva (FLEURY 1994; MENDES, SANCHES Y MOURA, 2009). ${ }^{10}$ Paralelamente, los derechos sociales, económicos y políticos pasan a ser traducidos en su formato más abstracto como derechos humanos, siendo "defendidos" por este tipo de política pública para los sectores más indigentes (COMPARATO, 1999).

La ciudadanía invertida indica ese reconocimiento "externo" de derechos, imputados por el Estado. Y la contrapartida de tal imputación es el conocimiento, reconocimiento y gestión minuciosos de la pobreza una pobreza cada vez más indigente y pasiva políticamente. Para ello, son necesarias nuevas formas de conocimientos y reconocimiento de la topografía humana de estos sectores poblacionales, como ya fue visto en las expresiones altamente tecnificadas de los PTC. Al reconocimiento abstracto de derechos le corresponde también el manejo también formal y abstracto de los procesos sociales. Paradójicamente, al reconocimiento e imputación, por parte del Estado, de derechos para las masas excluidas, le corresponde conocer y reconocer quiénes son los que los ostentan, a quiénes se les atribuye derechos, por qué razones y por cuánto tiempo. En definitiva, no se trata de derechos, sino del simple acceso y permanencia en este régimen asistencial de prestaciones provisorias (DE MARTINO, 2012).

\section{La ausencia de pluralidad en términos de familias y género}

Si bien los PTC apuntan a la población pobre e indigente (y, en nuestro país, a un universalismo por cierto limitado y de nueva era), a la hora de definir sus beneficiarios no hacen distinción entre los arreglos familiares que podrán acceder a las prestaciones. Incluso colocan especial énfasis en las familias monoparentales o "jefeadas" por mujeres. ¿Pero esto alcanza para indicar que los PTC poseen una definición amplia de familia y una perspectiva democrática de género? En un primer nivel de análisis, podríamos señalar que familias monoparentales o aquellas extendidas, podrían ser abordadas como prolongación de la familia nuclear en un caso, y como familia nuclear considerada incompleta en el otro, pues la función de paternaje estará presente de otra manera. Los entornos de la familia nuclear son los conocidos y reconocidos. En un segundo nivel, indicamos que la sociología de la familia debe ver más allá de la alianza y el parentesco, dos ejes que marcan la mirada sobre la familia. En esta oportunidad, múltiples preguntas pueden realizarse a los PTC. ¿Qué sucedería si tomamos como definición no a la familia sino a todo tipo de arreglo afectivo/sexual o como aquel núcleo con el cuál la persona cuenta en momentos buenos y malos (DE MARTINO, 2000)? Si la respuesta se caracteriza por la audacia, los PTC deberían incluir las familias homoparentales, a aquellos arreglos

${ }^{10}$ Sobre tales tipos de ciudadanía ver Fleury (1994); Mendes, Sanches y Moura (2009). 


\section{Revigta 미 paltg}

\} LOS PROGRAMAS DE TRANSFERENCIA DE RENTA - BERMÚDEZ, M. M.; GARRIDO, L. V. \}

DOI: $10.12957 /$ rep.2015.21052

constituidos por lazos de amistad y no de sangre o inclinación sexual. ¿Dos amigas y sus hijos viviendo juntas para abaratar los costos de la reproducción social, son familia o solo dos amigas buscando una salida para la sobrevivencia? ¿Por qué las políticas sociales continúan ciegas a aquellos referentes afectivos con gran carga emocional y simbólica, pero que no pueden incluirse en lo que tradicionalmente se entiende como familia? Tales personas necesitan de una nueva resignificación, no solo a la hora de la implementación de programas, sino a la hora de su diseño. Hablamos de una resignificación política, democratizadora e históricamente moderna.

Pero vayamos hacia una tercera línea de interpretación, tal vez complementaria. Una línea que desnuda, a la vez, la funcionalidad conservadora y modernizadora de la familia. Los PTC miran hacia el individuo, devenido en ciudadano como ya vimos, que atraviesa determinado ciclo de vida. Las prestaciones continúan teniendo como marco a la familia. Martínez (2005) nos dice que la familia opera como refuerzo, mecanismo de equilibrio y de reproducción, de las fuerzas asimétricas a partir de las cuales Estado y el mercado incluyen o excluyen a sus integrantes. La familia introyecta las ausencias, olvidos y efectos no deseados de las políticas sociales. Desde una perspectiva de género, también produce y reproduce la división sexual del trabajo y de los cuidados. En otras palabras, la familia constituye un espacio del régimen de producción de bienestar en funcionamiento (SPOSATI, 2011). Ahora, desde una perspectiva eminentemente política e instrumental, si la desfamiliarización/mercantilización significa reducir las cargas familiares, parecería que los PTC no se inclinan por esta línea. Cuanto más precarias son las circunstancias de vida de la persona y de su familia, más especializadas y focalizadas las políticas, por ende, más azaroso su acceso a los programas y su permanencia ya que el cumplimiento de las prestaciones se torna en mínimo casi inaccesible (SPOSATI, 2011).

Más aún, parecería que las exigencias respecto a las contraprestaciones y sus manuales de procedimientos subrayan este hecho. Tomemos ejemplos de la casuística derivada de la intervención en el campo judicial. Nos referimos a la judicialización del proceso de acceso a los beneficios, en este caso, a las AFAM: Se exige la ratificación judicial de la tenencia de niños y adolescentes, la cual supone acceder a un servicio jurídico, y muchas veces el proceso se demora por dificultades y conflictos familiares (progenitor con domicilio desconocido, negativa de alguna de las partes a la ratificación de la tenencia, etc.). O simplemente pensemos en familias ubicadas en situaciones duras y varias veces heredadas de exclusión, en las cuales es casi impensable la posibilidad de llevar a cabo con éxito todos estos trámites judiciales. Esto no es todo: niños y adolescentes que reciben el beneficio pueden dejar de hacerlo si la madre, por ejemplo, durante determinado mes, superó su nivel de ingresos realizando horas extras. Para volver a recibirlo, debe iniciar nuevamente el recorrido previsto. Si analizamos comparativamente, en nuestro país no se ha llevado a cabo 


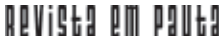

\} LOS PROGRAMAS DE TRANSFERENCIA DE RENTA - BERMÚDEZ, M. M.; GARRIDO, L. V.\}

DOI: 10.12957/rep.2015.21052

la definición del derecho a la asistencia como un derecho constitucional, lo que habilita a una judicialización de la asistencia pero en términos de demandas frente al no cumplimiento del estado de sus responsabilidades de protección no contributiva. Por el contrario, se ha judicializado el proceso de acceso a algunos beneficios pero no en términos de derechos, por formales que estos sean, sino a partir de exigencias para las familias beneficiarias.

Desde una perspectiva de género, los PTC no son innovadores. Se basan en una concepción de género binaria y respetuosa del mandato heterosexual. No se perciben a sí mismos como integrantes de un orden de género ni como regímenes de género (CONNELL, 1987). Por el contrario, la bibliografía acumulada indicaría que más bien reproducen las desigualdades de género. Respecto a esto, comenzamos por algo fundamental y que lo colocamos de manera puntual - la necesidad de investigaciones que permitan lo que destaca Daeren (2004): “(...) indagar acerca de cuáles son las oportunidades que tienen las mujeres que no están en el target de los programas, que no son madres, no se ubican en edad reproductiva o participan de familias no tradicionales" (SERRANO, 2005, p. 58). Es decir, que permitan reconocer el campo de los absolutamente excluidos, aún de los PTC. No olvidemos que tal vez esta invisibilidad pueda deberse al tipo de manejo estadístico y conceptual de la información, como ya hemos visto up supra: los promedios pueden dejar de lado parte de la población que necesita de tales programas.

Paralelamente, podrían fomentar sentimientos habitualmente considerados como femeninos, pero sentimientos provocados en mujeres para las cuales la ciudadanía no tiene aún significado, lo que evidenciaría un punto débil o un fracaso constatado de los PTC: "Estudios cualitativos realizados en el marco del programa Bono Solidario indican que este no tiene para las mujeres las connotaciones de un ingreso propio, ni de un derecho. Frente a la posibilidad de ser o no beneficiarias aparece un sentimiento de gratitud-resignación (Armas, 2004)" (SERRANO, 2005, p. 58).

Teniendo aún en vista solamente la distinción hombre/mujer cuando hablamos de género, cabe indicar que crece la jefatura femenina, lo que abre todo un campo de indagación: ¿la responsabilidad uniparental puede o no garantizar ciertos cuidados familiares de protección sin recargar a las mujeres de responsabilidades y actividades? En segundo lugar, parecería que los PTC establecen una remuneración al trabajo de la mujer como cuidadora del hogar, más allá de ser trabajadora formal o informal (SPOSATI, 2011). El papel de la mujer, como mediadora entre las demandas de la dinámica familiar y las del Estado, la sobrecarga de tareas que soporta o su condición de "pobre merecedora" han llevado a intensificar propuestas de desfamiliarización a partir de ciertos programas sociales. No obstante ello, la mujer parece ser la referente para las acciones estatales, lo que subraya la división sexual del trabajo y de los cuidados tanto a nivel material como simbólico. 


\section{Revigta 미 paltg}

\} LOS PROGRAMAS DE TRANSFERENCIA DE RENTA - BERMÚDEZ, M. M.; GARRIDO, L. V. \}

DOI: 10.12957/rep.2015.21052

Es decir, hablamos de un reforzamiento de los roles de género tradicionalmente atribuidos a las mujeres. Se señala que "la mayoría de los programas sitúa como perceptora de la transferencia a las mujeres, teniendo en cuenta que ellas son mejores administradoras de los recursos al interior del hogar y tienen mayor compromiso con la educación de los hijos" (SERRANO, 2005, p. 57). Hay quienes visualizan un mayor empoderamiento de la mujer en su carácter de preceptora pero los fundamentos sobre los que este carácter reposa refuerzan los estereotipos culturales referidos al ser hombre y al ser mujer: somos las mujeres las principales administradoras de la vida cotidiana del hogar y la crianza de los hijos y, por ello, en ellas se coloca la responsabilidad por el cumplimiento de las contrapartidas ${ }^{11}$.

Centrados en un patrón familiar que circunvala la familia nuclear y sus alrededores estadísticamente más reconocidos, parecería que los PTC, en lo que refiere a estas esferas de lo social, no han probado aún estar a la altura de las transformaciones de la contemporaneidad.

\section{A modo de cierre - Algunos efectos no deseados de los PTC}

A continuación, indicamos brevemente algunos efectos no deseados de los PTC a modo de síntesis del capítulo. Se trata de una breve enumeración de aspectos problemáticos que deben ser necesariamente transformados en objeto de reflexión.

1. La intersectorialidad en cuestión. Los defensores de los PTC valoran su propósito de articular un programa focalizado con servicios universales de educación y salud, lo cual es señalado como una innovación en los dispositivos de protección social ensayados hasta el momento. Son insuficientes aún los estudios que indagan acerca de la capacidad de los servicios educativos y de salud de hacer frente al incremento de la demanda que habría de esperar con el cumplimiento de las contrapartidas. Este punto coloca en cuestión otra característica de los PTC: su intersectorialidad. No existe, como ya dijimos, investigaciones que avancen en esta línea problemática (DE MARTINO, 2014). Asimismo, las evaluaciones señalan un efecto positivo de los PTC sobre la asistencia a centros educativos pero poco dicen acerca de la calidad del proceso formativo. Estos estudios no informan sobre los conflictos en la gestión cotidiana de los centros educativos y su posible relación con el ingreso de un universo poblacional que antes le era ajeno, así como la falta de reestructuración del sistema educativo para responder a las demandas de aumento de cobertura y las peculiares características, sobre todo a nivel secundario, de esta población adolescente y pobre. Las investigaciones existentes sobre este tópico coinciden en marcar

\footnotetext{
11 “Estos programas sitúan, una vez más, a las mujeres como 'intermediarias del bienestar' o de la 'economía del cuidado', que deposita en la mujer la responsabilidad de la atención de su grupo familiar con claras implicancias en su manejo del tiempo, sobrecarga de trabajo, tensiones y responsabilidades" (SERRANO, 2005, p. 58).
} 


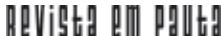

\} LOS PROGRAMAS DE TRANSFERENCIA DE RENTA - BERMÚDEZ, M. M.; GARRIDO, L. V.\}

DOI: 10.12957/rep.2015.21052

el aumento de la matriculación al sistema educativo y agregan que los efectos en la disminución de las tasas repetición, promoción y deserción son "modestos" y no producen efectos positivos en el nivel de aprendizaje (DRAIBE; RIESCO, 2009, p. 49).

2. Impactos débiles en el mundo del trabajo. Es importante destacar que de la evaluación intermedia del Panes surge que no se identifican efectos sobre las tasas de actividad, empleo y desempleo para titulares y que "se encuentra algún indicio de efecto negativo sobre la tasa de actividad masculina" (AMARANTE, BURDÍN, VIGORITO, 2007). Asimismo, no se observan efectos sobre la formalidad laboral para los titulares al tiempo que se señalan indicios de desincentivos a la formalidad en Montevideo y para otros integrantes del hogar (AMARANTE, BURDíN, VIGORITO, 2007). Es altamente probable que esta situación derive de la necesidad de los hogares de poseer los atributos que los vuelven posibles beneficiarios, siendo los ingresos uno de los indicadores tomados y fácilmente rastreables en condiciones de formalidad laboral. Se trata de un efecto no deseado de las decisiones definicionales sobre la exclusión - inclusión de la población por criterios de clasificación. Parecería que los programas que buscan la inserción en el mercado de trabajo no han tenido éxito, o que lo que se procuraba evitar - ver la prestación como un ingreso estable tipo salario -, se ha cumplido. De ser así, la racionalidad de los PTC se da de bruces con una otra racionalidad, apegada a la sobrevivencia, por parte de los beneficiarios. La descontextualización socio-cultural de la racionalidad, ya señalada en otro ítem, tal vez se encuentre asociada a este efecto no deseado.

3. Las hipótesis no corroboradas. El conjunto de PTC implementados en América Latina propone, como objetivos, aliviar la situación de indigencia y pobreza - asociada a múltiples riesgos - e interrumpir su ciclo de reproducción generacional a través del incremento del capital humano. Estos propósitos se fundan en hipótesis explicativas y en supuestos que resultan, al menos, discutibles. Las bondades y logros supuestos de estos programas se sustentan en hipótesis no fundadas (BARBA; VALENCIA, 2011, p. 201-202): no se ha demostrado que los PTC logren disminuir la pobreza, tampoco se ha comprobado su impacto esperado en la reducción del trabajo infantil y adolescente, ni que la exigencia de contrapartidas tengan como resultado una mejoría en el nivel educativo y tampoco se observan resultados auspiciosos en términos de ruptura del proceso de reproducción generacional de la pobreza: ${ }^{12}$ "Aun así, estos programas parecen estar siendo consolidados como pilar de las estrategias nacionales de enfrentamiento de la pobreza en la región" (DRAIBE; RIESCO, 2009, p. 39) En este punto hacemos alusión al referencial teórico de estos programas, la laxitud de sus supuestos, que ya han merecido nuestra atención.

12 "Sin embargo, los resultados de los PTCs son extremamente modestos en casi todas las dimensiones de sus objetivos, sean éstos la eliminación de la pobreza o los resultados en educación, salud y nutrición de los beneficiarios, como muestran las evaluaciones que comentamos en este estudio" (DRAIBE; RIESCO, 2009, p. 39). 


\section{Revigta 미 paltg}

\} LOS PROGRAMAS DE TRANSFERENCIA DE RENTA - BERMÚDEZ, M. M.; GARRIDO, L. V. \}

DOI: $10.12957 /$ rep.2015.21052

4. La estigmatización de los beneficiarios. La estigmatización de la población potencialmente beneficiaria se consagra inevitablemente cuando, desde la ingeniería política de estos dispositivos, se recurre a ciertos "valores implícitos en la consideración de las contrapartidas obligatorias; esto es, lo que supone atribuirles a las familias - y muy especialmente a los jefes de hogar -, negligencia, desidia o falta de interés en la educación y salud de sus hijos o hijas" (MIRZA, LORENZELLI; BANGO, 2010, p. 86). Asimismo, algunos mecanismos de control del cumplimiento de las contrapartidas son vejatorios de la intimidad de las familias, son tutelares e infantilizadores de la población beneficiaria. Este efecto no deseado es consecuencia directa de la necesidad de incorporar a los beneficiarios en un campo documental materializada en el desarrollo y en la sofisticación de sistemas de información de los organismos públicos que permitan controlar la correcta focalización de la prestación así como el cumplimiento de las condicionalidades a ella asociadas. Del mismo modo, los "cambios conductuales" deseados son reforzados por mensajes moralizantes y prescriptivos dirigidos a familias e individuos. El discurso moralizante apunta hacia la responsabilidad personal y al hacerse cargo de la propia vida (SCHMIDTZ; GOODIN, 2000).

5. La mercantilización de la asistencia. En el límite difuso entre impactos no deseados y decisiones en torno a definiciones se ubica la mercantilización de la asistencia materializada. Valga, a modo de ejemplo, el uso de las tarjetas magnéticas para la compra de alimentos dirigidas a la población más pobre dentro del PES. Este mecanismo sustituyó al sistema tradicional de canastas de alimentos provistas por el Instituto Nacional de Alimentación (Inda). Un argumento central de tal modificación fue el incremento de la libertad individual que los beneficiarios adquirían como consumidores cuando, mediante el uso de la tarjeta, podían seleccionar sus compras. Esta transformación ha sido objeto de preocupación por parte de las autoridades cuando se evidenció el crecimiento de la anemia por insuficiencia de hierro en niños menores de dos años. La anemia infantil había sido reducida con la introducción de leche fortificada con hierro en las antiguas canastas del Inda, pero al ser sustituidas por el uso de la tarjeta alimentaria, los beneficiarios optaban por comprar leche común, dado su menor costo. Los correctivos fueron puestos en andamiento ante una expresión de la transformación de los ciudadanos o quasi ciudadanos en consumidores o quasi consumidores.

6. El beneficiario desde un punto de vista filosófico. Por último, hablamos ya de ciertas miopías conceptuales de los PTC. Se encuentran atados a teorías que anulan una concepción de ser humano como síntesis de relaciones sociales incardinadas en cuerpos sexuados y en una identidad en tanto tales, de carácter universal y vinculada a las condiciones sociales de existencia. El abordaje de la naturaleza humana en su faz económica y supuestamente racional empobrece las propuestas y deja al desnudo la 


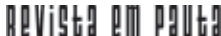

\} LOS PROGRAMAS DE TRANSFERENCIA DE RENTA - BERMÚDEZ, M. M.; GARRIDO, L. V.\}

DOI: 10.12957/rep.2015.21052

falacia del discurso basado en reconocimiento de derechos que podría definirse como el derecho a ser pobre, digno y asistido. Paralelamente ese ser pobre se manifiesta como sexuado de manera binaria, sin incorporar los matices de las diferentes opciones sexuales. Y si el ser humano se desarrolla junto a otros en ámbitos como la familia, en su carácter de grupo primario (HELLER, 1985), los PTC también son poco condescendientes con la pluralidad de arreglos familiares que pueden ser entendidos a partir de una definición amplia de familia, como grupo que facilita la reproducción, unido por lazos afectivos ligados o no al parentesco o alianza tradicionalmente entendida. En tal sentido, los PTC nos retrotraen a la figura del pobre merecedor, ya no hombre, tal vez mujer por las razones ya esgrimidas: blanca, heterosexual y dueña de una racionalidad aceptada y definida por la filantropía en el siglo XIX (DE MARTINO, 2012; 2013).

Más allá de buenas intenciones, los PTC dan la bienvenida a cierta refilantropización de la asistencia que augura una cultura política, si bien progresista, algo conservadora en sus propuestas (derechos en su expresión abstracta y formal; padrón heterosexual predominante; reforzamiento de roles tradicionales imputados a los sexos; estigmatización de los beneficiarios; fundamentos teóricos que hablan de una economización de lo social (DE MARTINO, 2009; 2012).

\section{Agradecimientos}

El artículo se enmarca en el Proyecto de Investigación titulado: Estudio comparado de Programas de Transferencia de Renta Condicionada en Brasil (Bolsa Família); Argentina (Asignación Universal por Hijo) y Uruguay (Asignaciones Familiares), financiado por la Comisión de Perfeccionamiento del Personal de Enseñanza Superior - CAPES -, del Ministerio de Cultura de Brasil, a partir del Programa PPCP - MERCOSUL, de acuerdo al Edital CGCI nº. 072/2010, Proceso Selectivo 2010/2011. Envolvió a los Programas de Posgrado en Políticas Públicas de la Universidad Federal de Maranhão/Brasil y el Programa de Doctorado en Ciencias Sociales - Mención Trabajo Social, de la Facultad de Ciencias Sociales de la Universidad de la República. Agradecemos a las instituciones e investigadores involucrados. 


\section{Revigta 미 paltg}

\} LOS PROGRAMAS DE TRANSFERENCIA DE RENTA - BERMÚDEZ, M. M.; GARRIDO, L. V. \}

DOI: 10.12957/rep.2015.21052

\section{Referencias}

AMARANTE, V.; BURDÍN, G.; VIGORITO, A. Primeros resultados de la evaluación intermedia del PANES. Montevideo, Instituto de Economía, Facultad de Ciencias Económicas, Universidad de la República. Disponible en: <http://www.mides.gub.uy/innovaportal/file/6570/1/metodologia_ de_la_seleccion_de_la_poblacion_del_plan_de_atencion_nacional_a_la_emer gencia_social_panes.pdf.>. Acceso en: 11 abr. 2012 .

AMARANTE, V; ARIN, R.; VIGORITO, A. (s/f). Metodología para la selección de participantes en el Plan de Emergencia Social.

BANCO MUNDIAL. (s/f). Las políticas de transferencia de ingresos en Uruguay: cerrando las brechas de cobertura para aumentar el bienestar. Disponible en: <http://www.siteresources.worldbank.org/INTURUGUAYINSPA $\mathrm{NISH} /$ Resources/PoliticasdeTransferencia.pdf>. Acceso en: 11 abr. 2011.

BARBA, C.; VALENCIA, E. Hipótesis no comprobadas y espejismos de las Transferencias Monetarias Condicionales. In: SOLANO, C.; COHEN, N. (coord.) Perspectivas críticas sobre la cohesión social. Desigualdad y tentativas fallidas de integración social en América Latina. Buenos Aires: Consejo Latinoamericano de Ciencias Sociales . 2011.

BECKER, G. Social choice and individual values. New Haven: Yale University Press. 1976.

BURCHARD, H. El Nuevo combate internacional contra la pobreza. Nueva Sociedad. Disponible en: <http://www.plataformademocratica.org/Publica coes/22964_Cached.pdf.> Acceso en: 13 oct. 2013.

CASTEL, R. La gestión de los riesgos. De la antipsiquiatría al post - análisis. Anagrama: Barcelona. 1984.

CASTELLS, M. A sociedade em rede. São Paulo: Paz e Terra.1999.

COLEMAN, J. Foundations of social theory. Cambridge: Harvard University Press. 1990.

COMPARATO, F. A afirmação histórica dos direitos do homem.São Paulo: Saraiva. 1999.

CONNELL, R. Gender and power. Society, the person and sexual politics. California: Stanford University Press. 1987.

DE MARTINO, M. Familias, género e integración regional. Un ejemplo en el Mercosur: la ciudad Rivera - Sant'Ana do Livramento. Campinas: Unicamp. 2000.

. La economización de lo social. U. A. H., Santiago de Chile: Perspectivas, n. 20. 2009. 


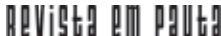

\} LOS PROGRAMAS DE TRANSFERENCIA DE RENTA - BERMÚDEZ, M. M.; GARRIDO, L. V.\}

DOI: 10.12957/rep.2015.21052

. Estudio comparado de Programas de Transferencia de Renta Condicionada en Uruguay - Argentina y Brasil. In: SEGUNDA MISIÓN DE TRABAJO, Montevideo. 2012.

. (coord.) Estudio comparado de Programas de Transferencia de Renta Condicionada en Uruguay - Argentina y Brasil. In: CUARTA MISIÓN DE TRABAJO, Tandil. 2013.

. Políticas sociales, familias y profesiones asistenciales. Algunos puntos actuales de tensión. In: AA.VV. Parentalidades y cambios familiares. Enfoques teóricos y prácticos. Montevideo: Inau. 2014.

DE MARTINO, M.; VECINDAY, L. Las nuevas formas de gobierno poblacional. Sobre individualización e ingeniería social. Fronteras, número especial, Montevideo: Departamento de Trabajo Social - Facultad de Ciencias Sociales. 2011.

DRAIBE, S. Brasil: Bolsa-Escola y Bolsa-Familia. In: COHEN, E FRANCO, R. (coord.) Transferencias con corresponsabilidad. Una mirada latinoamericana. México: Facultad Latino Americana de Ciencias Sociales. 2006.

DRAIBE, S.; RIESCO, M. El Estado de bienestar social en América Latina. Una nueva estrategia de desarrollo. Documento de Trabajo $n^{\circ} 31$. Madrid: Fundación Carolina, CeAlci. 2009.

DONZELOT, J. A policía da familia. Rio de Janeiro: Graal. 1986.

FILGUEIRA, F.; MOLINA, G.; PAPADÓPULOS, J.; TOBAR, F. Universalismo básico: una alternativa posible y necesaria para mejorar las condiciones de vida en América Latina. Instituto Interamericano para el Desarrollo Social (INES).2006.Disponible en: <www.inau.gub.uy/biblioteca/universalismo .pdf.> Acceso en: 24 nov. 2012.

FLEURY, S. Estado sem cidadãos. Rio de Janeiro: Fiocruz. 1994.

FRASER, N. lustitia interrupta. Reflexiones críticas desde la posición "postsocialista" Santiago de Bogotá: Siglo del Hombre Editores. 1997.

GRASSI, E. Política y problemas sociales en la sociedad neoliberal. La otra década infame (I). Buenos Aires: Espacio Editorial. 2003.

HELLER, A. Historia y vida cotidiana. México: Editorial Grijalbo. 1985.

HOLZMANN, R.; JORGENSEN, S. Manejo social del riesgo: un nuevo marco conceptual para la protección social y más allá. Revista de la Facultad Nacional, Colombia: Universidad de Antioquia, v. 21, n. 1. 2003.

IVO, A. Bolsa Família: caminhos de formação da coesão social ou segmentação da pobreza? In: SOLANO, C.; COHEN, N. (coord.) Perspectivas críticas sobre la cohesión social. Desigualdad y tentativas fallidas de integración social en América Latina. Buenos Aires: Consejo Latinoamericano de Ciencias Sociales. 2011. 


\section{Revigta 미 paltg}

\} LOS PROGRAMAS DE TRANSFERENCIA DE RENTA - BERMÚDEZ, M. M.; GARRIDO, L. V. \}

DOI: $10.12957 /$ rep.2015.21052

LAWSON, T. Abstraction, tendencies an stylised facts: a realist approach to economic analysis. Cambridge Journal of Economics, n. 13. 1990

MARTÍNEZ BARROSO, M. La asistencialización de la Protección Social. Tribuna social: revista de seguridad social y laboral, Valencia: Praxis, n. 169. 2005.

MARTÍNEZ, J. Regímenes de bienestar en América Latina: consideraciones generales e itinerarios regionales. Revista Centro-Americano de Ciencias Sociales, San José de Costa Rica: Flacso, v. 2, n. 2. 2005.

MENDES, J.; SANCHES, D.; MOURA, M. Proteção social e a saúde do trabalhador: contingências do sistema de mediações sociais e históricas. Revista Políticas Públicas, Maranhão: Universidade Federal do Maranhão, v. 13, n. 1. 2009.

MIDAGLIA C., SILVEIRA M. Políticas sociales para aminorar el conflicto social y enfrentar los desafíos de la cohesión social. Los nuevos programas de Transferencias Condicionadas de Renta en Uruguay. In: SOLANO, C.; COHEN, N. (coord.) Perspectivas críticas sobre la cohesión social. Desigualdad y tentativas fallidas de integración social en América Latina Buenos Aires: Consejo Latino Americano de Ciencias Sociales. 2011.

MIRZA, C. Experiencias y desafíos de implementación de una nueva malla de protección social, el caso del Plan de Equidad. In: SERNA, M. (coord.) Pobreza y (des)igualdad en Uruguay: una relación en debate. Montevideo: Consejo Latinoamericano de Ciencias Sociales. 2010.

MIRZA, C.; LORENZELLI, M.; BANGO, J. (coord.) ¿Es posible un nuevo Estado de Bienestar en América Latina? La reconfiguración de las matrices de bienestar en el MERCOSUR. Avances de Investigación. Montevideo: Fundación Carolina. 2010.

MUÑOZ, C. Uruguay homosexual. Culturas, minorías y discriminación desde una sociología de la homosexualidad. Montevideo: Ediciones Trilce. 1996

OLSON, M. The logic of collective action. Cambridge: Harvard University Press. 1965.

PAZ, J. Programas dirigidos a la pobreza en América Latina y el Caribe. Sustento teórico, implementación, práctica e impactos sobre la pobreza en la región. Buenos Aires: Consejo Latino Americano de Ciencias Sociales. 2010.

SANTOS, B. de Sousa. Para descolonizar Occidente: más allá del pensamiento abismal. Buenos Aires: Facultad de Filosofía y Letras. 2010.

SCHMIDTZ, D.; GOODIN, R. El bienestar y la responsabilidad individual. Madrid: Cambridge University Press. 2000. 


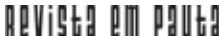

\} LOS PROGRAMAS DE TRANSFERENCIA DE RENTA - BERMÚDEZ, M. M.; GARRIDO, L. V.\}

DOI: 10.12957/rep.2015.21052

SERNA, M. Las políticas de la pobreza en los gobiernos de izquierda del Cono Sur: de las redes sociales de seguridad a las redes de protección social. Las políticas sociales de las izquierdas ¿un modelo de desarrollo alternativo? Buenos Aires: Clacso. 2007.

. La evolución del sistema de Seguridad Social en el Uruguay (18291986). Montevideo: Instituto de Economía (UdelaR). 2007.

SERRANO, C. La política social en la globalización. Programas de protección en América Latina. Serie Mujer y Desarrollo. Santiago de Chile: Cepal. 2005.

SOUSA, J. de; Análise das condições de desenvolvimento familiar em Niterói: uso do IDF e Gini. Revista de Políticas Públicas, Maranhão: Universidade Federal do Maranhão, v. 14, n. 2. 2010.

SPOSATI, A. Tendências latino-americanas da política social pública no século 21. Revista Katálysis. Políticas sociais e questões contemporâneas, Florianópolis: Editora UFSC, v. 14, n. 1. 2011.

VIGORITO, A., ARIM, R.; SALAS, G.; AMARANTE, V. Desarrollo económico y desigualdad en Uruguay. In: SERNA, M. (coord.) Pobreza y (des)igualdad en Uruguay: una relación en debate. Montevideo: Clacso. 2011.

Recebido em 19 de julho de 2015.

Aprovado para publicação em 12 de dezembro de 2015.

DOI: 10.12957/rep.2015.21052

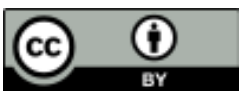

A Revista Em Pauta: Teoria Social e Realidade Contemporânea está licenciada com uma Licença Creative Commons Atribuição 4.0 Internacional. 